\title{
A decision-making model for selecting the most appropriate natural fiber - polypropylene-based composites for automotive applications
}

\begin{abstract}
Recent emphasis on the proper utilization of the available natural resources as well as the compatibility between industrial sustainability and the environment have recognized the natural fiber reinforced polymer composites as a valuable type of materials. The final features of natural fiber reinforced polymer composites are strongly influenced by the characteristics of their constituents. Several factors affect implementing natural fiber reinforced polymer composites in automotive applications, which make it a matter of multi-criteria decision making problem. In this work, a decision-making model is introduced to evaluate and select the most appropriate non-woven natural reinforcement fiber/polypropylene-based composites for interior parts in the automotive industry considering various evaluation criteria simultaneously. The analytical hierarchy process was utilized to assess 15 potential alternatives of different natural fiber/polypropylene composites. The evaluation process considered simultaneous six evaluation criteria, namely tensile strength, tensile modulus, flexural strength, flexural modulus, impact strength and the maximum water absorption of the composites. Such approach is implemented here for the first time to enhance the selection process of natural fiber reinforced polymer composites for automotive application and to expand the future sustainable design possibilities in this sector. Both impact and flexural strengths were found to have the major priority in the evaluation process. The treated flax/polypropylene composite with $30 \mathbb{t}$ t\% fiber loading alternative was found to be the best with regard to the overall evaluation criteria. Date palm fiber/polypropylene composites were found to be potential considering the whole desired evaluation criteria.
\end{abstract}

Keyword: Automotive industry; Bio-based composites selection; Date palm fibers; Decisionmaking model; Polypropylene; Sustainable products 